\title{
Inner dynein arm defects causing primary ciliary dyskinesia: repeat testing required
}

\author{
C. O'Callaghan, A. Rutman, G.M. Williams and R.A. Hirst
}

\begin{abstract}
Primary ciliary dyskinesia (PCD) results in chronic nasal symptoms and chest disease leading to bronchiectasis. We noted a number of patients referred for diagnostic testing whose initial results suggested PCD due to an inner dynein arm or radial spoke defect but in whom no abnormality was found on retesting.

The present study was an audit of all patients referred for PCD diagnostic testing over a 3-yr period whose initial electron microscopy (EM) and beat pattern analysis suggested an inner dynein arm or radial spoke defect.

21 patients referred for diagnostic testing for PCD suspected of an inner dynein arm defect and six suspected of a radial spoke defect on initial EM and beat pattern analysis had repeat testing performed. On repeat testing, five patients initially suspected of an inner dynein arm defect and one with a radial spoke defect had normal EM and beat pattern, leading to the initial diagnosis being questioned.
\end{abstract}

Patients suspected of PCD due to an inner dynein arm defect or radial spoke defect should have the diagnosis reassessed if it has been based on only one diagnostic sample.

KEYWORDS: Bronchiectasis, cilia, inner dynein arm defect, primary ciliary dyskinesia, radial spoke defect

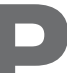

rimary ciliary dyskinesia (PCD) is a congenital disorder with a prevalence of between 1:2,500 and 1:30,000 live births

[1-3]. It causes impaired mucociliary clearance due to motionless or dyskinetically beating cilia, leading to chronic upper and significant lower respiratory tract disease [4-6]. Specialist diagnostic testing is required and the diagnosis of $\mathrm{PCD}$ can be difficult [7].

PCD is associated with a number of ultrastructural defects of the ciliary axoneme that result in defective ciliary function [4] and specific ultrastructural defects have been shown to produce characteristic ciliary beat patterns [8]. Currently, two diagnostic techniques - the analysis of ciliary beat pattern [9] and frequency, and transmission electron microscopy (EM) analysis - are recommended as the principal diagnostic tests for patients suspected of PCD [4].

However, assessment of ciliated epithelial samples can be difficult, particularly if there is underlying epithelial damage. Even in cilia from healthy ciliated epithelium, EM analysis can be problematic, particularly for the analysis of inner dynein arms because of their low contrast on EM [10].

Because of concerns about the possibility of falsepositive diagnoses, we repeated diagnostic testing of cases where a positive diagnosis of PCD was suggested from analysis of ciliary function and EM on the initial brush biopsy. We noted that occasionally when an inner dynein arm defect was suspected on EM and functional analysis, and a subsequent sample was taken, ciliary function and EM were normal, thus excluding the diagnosis. This was also noted in a patient who was suspected of having a radial spoke defect on an initial sample. A radial spoke defect is a combination of an inner dynein arm defect with disarrangement of the peripheral and central microtubules of the ciliary axoneme. In contrast, in patients whose initial sample suggested PCD due to either an outer dynein arm defect, a combined inner and outer dynein arm defect, or a transposition defect, repeat testing confirmed the diagnosis in each case. Herein, we audit the patients referred to our PCD diagnostic centre over the past 3 yrs where an inner dynein arm defect or radial spoke defect was suspected on examination of the first epithelial brush biopsy, in order to determine the proportion of cases where the diagnosis was excluded on subsequent biopsies.

\section{METHODS}

The audit consisted of patients referred to one of the UK National Diagnostic Centres for PCD (Leicester, UK) over a 3-yr period, where a

\section{AFFILIATIONS}

Division of Child Health \& Institute of Lung Health, Dept of Infection, Immunity and Inflammation, University of Leicester, Leicester, UK.

\section{CORRESPONDENCE}

C. O'Callaghan

Dept of Infection, Immunity

and Inflammation

University of Leicester

Robert Kilpatrick Clinical

Sciences Building

PO Box 65

Leicester Royal Infirmary

Leicester

LE2 7LX

UK

E-mail: ajb64@le.ac.uk

Received:

July 122010

Accepted after revision:

Feb 142011

First published online:

March 152011 
diagnosis of an inner dynein arm defect or a radial spoke defect was strongly suspected from analysis of the initial biopsy.

An inner dynein arm defect was defined as a complete absence of all inner dynein arms or presence of all inner dynein arms as very short stub-like projections as opposed to the fully developed arm. In a radial spoke defect, again there is an absence of inner dynein arms associated with disarrangement of the microtubules within the ciliary axoneme, allowing the displacement of one of the outer doublets inwards towards the central pair.

Ciliated samples were obtained by brushing the nasal epithelium with a 2-mm cytology brush (Olympus Keymed Ltd, Southendon-Sea, UK). At the time of nasal brush biopsy, all patients reported that they had not had a symptomatic acute upper respiratory tract infection for at least 6 weeks.

Ciliary beat frequency and ciliary beat pattern analysis were performed as described previously using a digital high-speed video camera (Kodak Motioncorder Analyser 1000; Eastman Kodak, Rochester, NY, USA) [8, 11] or the Motion Pro X4 digital high-speed video camera (Lake Image Systems, Henrietta, NY, USA).

\section{Ciliary ultrastructure assessment}

Samples were processed for transmission EM for ultrastructural analysis of the ciliary axoneme $[12,13]$. In summary, EM sections were examined in a methodical grid square search. The health and condition of cells was noted at low power $(\times 5,000)$. Cells were then observed under medium power $(\times 66,000)$ to assess for the quality of the cross-sections. Sections that had been cut in the best alignment were suitable for microtubular and dynein arm assessment. A high-power examination $(\times 250,000)$ of the cilia from each cell was assessed for the presence and absence of dynein arms and for microtubular structure and arrangement. Data were recorded on all of the cross-sections seen. Whenever possible, at least 10 individual cells were studied. The percentage of cilia with dynein arm microtubular defects was recorded [12].

Ciliary function assessment was blinded to the results of EM analysis and vice versa, as is the standard practice in our referral centre.

\section{Nasal nitric oxide measurement}

Nasal nitric oxide (nNO) was measured using a chemiluminescence analyser (Sievers 280i; Analytix, Boldon, UK) with a transnasal flow rate of $2 \mathrm{~L} \cdot \mathrm{min}^{-1}$, following the American Thoracic Society/European Respiratory Society guidelines [14]. nNO was measured in patients attending the Leicester clinic who had patent airflow across the nasopharynx and, due to difficulty of the restricted exhaled breath manoeuvre, were aged $>5$ yrs [14].

\section{Air-liquid interface cell culture}

The methodology for air-liquid interface cell culture has been described in detail previously [15]. Briefly, nasal brush biopsy samples were grown on collagen-coated $(0.1 \%$; Vitrogen, Leimuiden, The Netherlands) 12-well tissue culture trays in Bronchial epithelial growth media (BEGM; Lonza, Portsmouth, NH, USA) for 7-14 days. The basal cells were seeded on a collagen-coated 12-mm diameter transwell clear insert (Costar; Corning, Lowell, MA, USA) under BEGM for 2 days. The basal cell monolayer was fed on the basolateral side only with ALImedia (50\% BEGM and 50\% Hi-glucose minimal essential medium containing $100 \mathrm{nM}$ retinoic acid). When cilia were observed on the cultures of difficult patients (with a high dyskinesia score on the original brush biopsy) or suspected PCD patients, they were physically removed from the transwell insert by gentle scraping with a spatula and washing with $1 \mathrm{~mL}$ HEPES (20 mM)-buffered medium 199 containing penicillin $\left(50 \mathrm{~g} \mathrm{~mL}^{-1}\right)$, streptomycin $\left(50 \mathrm{~g} \mathrm{~mL}^{-1}\right)$ and Fungizone $\left(1 \mathrm{~g} \mathrm{~mL}^{-1}\right)$. The recovered ciliated epithelium was then dissociated by gentle pipetting. $100 \mu \mathrm{L}$ of the cell suspension was placed in a microscope chamber slide and ciliary beat frequency and pattern were assessed as described previously. The remaining $900 \mu \mathrm{L}$ was fixed in glutaraldehyde for transmission EM analysis of the axoneme structure. In the authors' clinic (Dept of Infection, Immunity and Infection, University of Leicester, Leicester, UK), cell culture is performed in all samples that are suspected of having PCD [15].

\section{RESULTS}

Over a 3-yr period, 724 patients were referred for diagnostic testing for PCD. Of them, 21 were suspected of PCD due to an inner dynein arm defect and six due to a radial spoke defect on initial sampling. Repeat diagnostic sampling of these patients, on one further occasion, did not confirm the diagnosis in six $(22 \%)$ cases. The confirmed radial spoke and inner dynein arm cases are described below, followed by a more detailed description of the cases where a repeat biopsy gave normal results, excluding the diagnosis of PCD.

We had previously noted that although some patients with suspected inner dynein arm defects and radial spoke defects showed no abnormalities on retesting, this was not the case for other common phenotypes of PCD. Because of this, not all of the patients with other defects were retested during the study period reported in this paper. However, 27 patients diagnosed with other PCD phenotypes (18 with an outer or outer plus inner dynein arm defect out of a total of 52 patients diagnosed with these defects; nine out of nine patients diagnosed with a transposition defect) had repeat biopsies that in each case confirmed the original diagnosis.

\section{Confirmed radial spoke defects}

Six cases of a radial spoke defect were diagnosed, five in children (aged 1 month to 9 yrs) and one in a 60-yr-old. All children had daily respiratory symptoms from the first year of life and nasal symptoms from early infancy. Although all were born after 36 weeks' gestation, three had been admitted to a special care baby unit following delivery because of respiratory distress. One patient had glue ear causing hearing difficulties and two patients aged $<1 \mathrm{yr}$ of age were awaiting hearing assessment. Two patients had situs inversus. One of the patients with situs inversus also had transposition of the great arteries and a ventricular septal defect. The mean ciliary beat frequency was 8.6 Hz (range 3-13 Hz) on the first biopsy and $8.3 \mathrm{~Hz}$ (range 5$12.5 \mathrm{~Hz}$ ) on the second biopsy. nNO was low at 48,59 and 5 parts per billion in the three patients old enough to be tested. EM revealed an absence of inner dynein arms and disarrangement of the peripheral and central microtubular pairs, typical of a radial spoke defect, on both biopsies. 


\section{Confirmed inner dynein arm defects}

15 cases of an inner dynein arm defect were diagnosed in 14 children (aged 1-15 yrs) and one in a 30-yr-old. All had daily respiratory symptoms from the first year of life and nasal symptoms from early infancy. Although all were born after 36 weeks' gestation, six had been admitted to a special care baby unit following delivery because of breathing difficulties. 10 patients had glue ear causing hearing difficulties and five had had grommets inserted. Six patients had situs inversus. One patient also had a ventricular septal defect and polysplenia. The mean ciliary beat frequency was $10.4 \mathrm{~Hz}$ (range 4-17 Hz) on the first biopsy and $9.9 \mathrm{~Hz}$ (range 5-16 Hz) on the second biopsy. $\mathrm{nNO}$ was measured in 11 patients and in all but one patient ( $\mathrm{nNO}=142$ parts per billion) was below 50 parts per billion. EM revealed an absence of inner dynein arms on both biopsies. Cell culture to a ciliated phenotype was possible in eight cases confirming an absence of inner dynein arms and a stiff beat pattern of all cilia observed.

\section{Diagnosis of PCD unlikely on retesting}

In six cases, the initial sample suggested an inner dynein arm defect $(n=5)$ or a radial spoke defect $(n=1)$ on both beat pattern analysis and transmission EM. In four of the initial samples, the epithelium obtained was recorded as being unhealthy. The details of these patients are outlined in tables 1 and 2 .

\section{DISCUSSION}

In 27 patients out of 724 referred for diagnostic testing for PCD over a 3-yr period, the initial epithelial brush biopsy suggested a possible diagnosis of an inner dynein arm defect or a radial spoke defect. In all cases, the biopsy was repeated and in six (22\%) cases, five with suspected inner dynein arm defect and one with a radial spoke defect, the diagnosis was considered unlikely by the presence of a normal ciliary beat pattern and a normal ciliary ultrastructure on EM. These results strongly suggest that any patient suspected on initial testing of an inner dynein arm defect or an inner dynein arm defect associated with a radial spoke defect should have their diagnosis confirmed by repeat testing. In PCD due to an outer dynein arm defect, a combined inner and outer dynein arm defect or a transposition defect, repeat biopsies have proved identical to the initial sample on each occasion.

The reasons for the change in the initial beat pattern and EM phenotype are unclear. In four of the initial samples, the epithelium obtained was recorded as being unhealthy, but with enough ciliated edges to allow analysis to proceed. However, in two cases the epithelium appeared healthy. It is known that damage to the epithelium, for example following a viral upper respiratory tract infection, can affect ciliary function and the EM appearance of cilia [13, 16-18]. Although we do not biopsy patients who have reported a symptomatic upper respiratory tract infection within the previous 6 weeks, we know that a proportion of people with a viral infection may show significant damage to the ciliated respiratory epithelium without being aware of any symptoms [13].

Four of the initial samples had been couriered from peripheral units and examined within $8 \mathrm{~h}$ of the initial brushing. It was noted that the epithelium appeared unhealthy in these samples but sufficient ciliated edges were seen to allow analysis. We have previously observed that couriered samples may have a

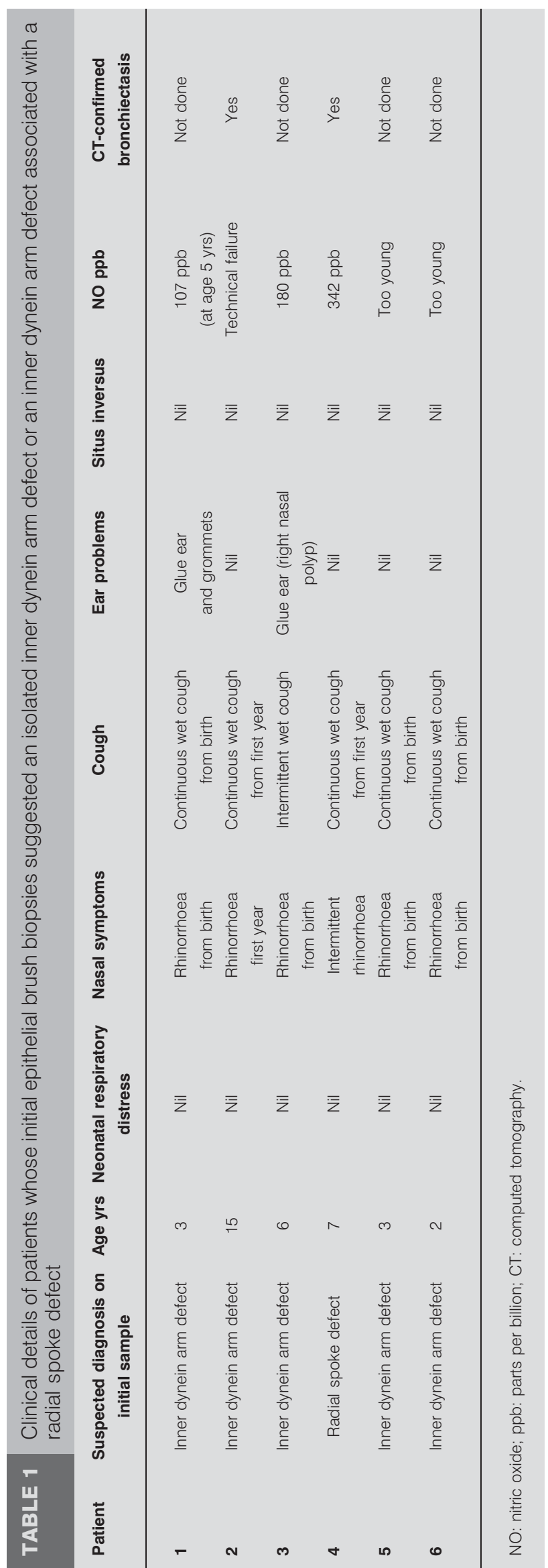

VOLUME 38 NUMBER 3 


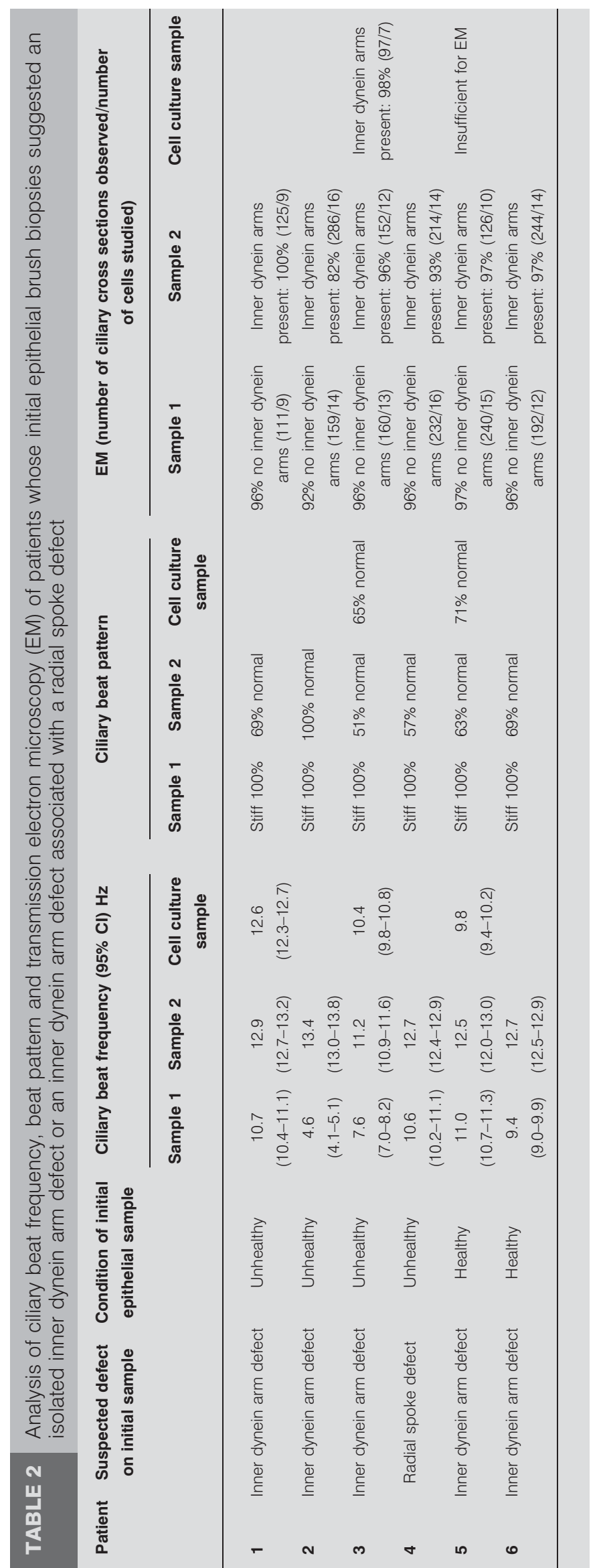

higher incidence of secondary ciliary dyskinesia [19] and it is possible that this may have contributed to our initial findings in two cases. However, two of the patients who were siblings and who were seen together at the diagnostic centre on both occasions were suspected of an inner dynein arm on the initial sample. The respiratory epithelium obtained on both occasions appeared healthy.

Cell culture to a ciliated phenotype was possible in eight cases with isolated inner dynein arm defects and in one case with a radial spoke defect. In all but one case culture confirmed the initial suspicion of the underlying ciliary defect. In one case, where an inner dynein arm defect was suspected on examination of the initial biopsy, ciliary structure and function of the cultured epithelium was normal. Cell culture, however, is a highly specialised technique and our success rate of culturing nasal ciliated epithelium from patients suspected of PCD using an air-liquid interface method is only $54 \%$ [15]. If cell culture results had been successful from all of the initial biopsies this would have proved helpful. Unfortunately, cell culture was successful on only one out of the five patients suspected of an inner dynein arm defect on their initial biopsy. When this patient's ciliated culture was examined, the ciliary beat pattern was found to be normal and inner dynein arms were clearly seen. This again suggests a secondary problem in the initial sample.

The clinical presentation of patients with PCD is well described in adults [6] and in children [9]. From the clinical history, diagnostic testing was strongly indicated in five out of the six patients whose repeat testing was normal. These patients had a history of a chronic wet sounding or productive cough from infancy and chronic nasal symptoms. The other patients had similar respiratory problems but only intermittent nasal symptoms. It is of interest, however, that none of these patients had situs inversus and none required admission to a special care baby unit because of respiratory distress following birth. In the group where the diagnosis was confirmed on retesting, situs inversus was observed in 33\% and $43 \%$ had been admitted to a special care baby unit because of respiratory distress after birth.

Various studies have shown that levels of exhaled nitric oxide, particularly $\mathrm{nNO}$, are very low in patients with $\operatorname{PCD}[6,20,21]$. None of the patients in whom the diagnosis of PCD was excluded on retesting had had their $\mathrm{nNO}$ measured when their initial sample was taken. One patient was too young and in one case there was a technical failure relating to the measurement. The remaining four samples were couriered from peripheral units where measurement of $\mathrm{nNO}$ is not available. When measured in three of the patients during repeat diagnostic testing, nNO was within the normal range, again suggesting the initial suspicion of PCD was incorrect [5]. However, a few patients with PCD have been found to have nitric oxide levels within the normal range [20]. Indeed, one of our patients with findings consistent of an inner dynein arm defect on initial and subsequent sampling had an $\mathrm{nNO}$ within the normal range.

We have not repeated the nasal brushing of the six patients in whom the diagnosis of PCD was initially suspected and later considered unlikely to determine whether dynein arms were again impossible to see and ciliary function suggested an inner 
dynein arm defect. Therefore, we are not able to comment on whether the inner dynein arms could be temporarily knocked down in certain situations, for example following a viral infection. These patients all had chronic respiratory symptoms and we do not know whether the inner dynein arm defect may be intermittent and reappear, representing an unusual ciliary phenotype.

It is acknowledged that inner dynein arms are more difficult to observe due to the decreased repeats along the ciliary axoneme compared to the outer dynein arms. EsCUDIER et al. [10] have suggested the use of computer-assisted analysis to help detect inner dynein arm abnormalities. However, such analysis is not widely used. Evaluation of this technique in other centres and the development of other methods to enhance visualisation of inner dynein arms are required.

As previously noted, the use of ciliary beat frequency alone to screen for biopsies requiring further assessment by electron microscopy may result in diagnoses being missed. This is true for a proportion of the patients in this study, where the beat frequency was $>11 \mathrm{~Hz}$, a cut-off previously suggested above which EM was not indicated [22].

In summary, the results of this audit suggest that patients in whom PCD has been diagnosed or is suspected due to an inner dynein arm defect alone, or an inner dynein arm defect combined with a radial spoke defect, should be retested to avoid the possibility of a false-positive diagnosis. We currently repeat diagnostic testing of patients who were suspected of an inner dynein arm defect or radial spoke defect and those who have an unusual phenotype of PCD and those whose initial biopsy shows significant secondary damage making interpretation difficult.

\section{STATEMENT OF INTEREST}

None declared.

\section{REFERENCES}

1 O'Callaghan C, Chetcuti P, Moya E. High prevalence of primary ciliary dyskinesia in a British Asian population. Arch Dis Child 2009; 95: 51-52.

2 Bush A, Chodhari R, Collins N, et al. Primary ciliary dyskinesia: current state of the art. Arch Dis Child 2007; 92: 1136-1140.

3 Kuehni CE, Frischer T, Strippoli MP, et al. Factors influencing age at diagnosis of primary ciliary dyskinesia in European children. Eur Respir J 2010; 36: 1248-1258.

4 Barbato A, Frischer T., Kuehni CE, et al. Primary ciliary dyskinesia: a consensus statement on diagnostic and treatment approaches in children. Eur Respir J 2009; 34: 1264-1276.

5 Marthin JK, Petersen N, Skovgaard LT, et al. Lung function in patients with primary ciliary dyskinesia: a cross-sectional and 3-decade longitudinal study. Am J Respir Crit Care Med 2010; 181: 1262-1268.

6 Noone PG, Leigh MW, Sannuti A, et al. Primary ciliary dyskinesia: diagnostic and phenotypic features. Am J Respir Crit Care Med 2004; 169: 459-467.

7 Papon JF, Coste A, Roudot-Thoraval F, et al. A 20-year experience of electron microscopy in the diagnosis of primary ciliary dyskinesia. Eur Respir J 2010; 35: 1057-1063.

8 Chilvers MA, Rutman A, O'Callaghan C. Ciliary beat pattern is associated with specific ultrastructural defects in primary ciliary dyskinesia. J Allergy Clin Immunol 2003; 112: 518-524.

9 Stannard WA, Chilvers MA, Rutman AR, et al. Diagnostic testing of patients suspected of primary ciliary dyskinesia. Am J Respir Crit Care Med 2010; 181: 307-314.

10 Escudier E, Couprie M, Duriez B, et al. Computer-assisted analysis helps detect inner dynein arm abnormalities. Am J Respir Crit Care Med 2002; 166: 1257-1262.

11 Chilvers MA, Rutman A, O'Callaghan C. Functional analysis of cilia and ciliated epithelial ultrastructure in healthy children and young adults. Thorax 2003; 58: 333-338.

12 Rayner CF, Rutman A, Dewar A, et al. Ciliary disorientation alone as a cause of primary ciliary dyskinesia syndrome. Am J Respir Crit Care Med 1996; 153: 1123-1129.

13 Chilvers MA, McKean M, Rutman A, et al. The effects of coronavirus on human nasal ciliated respiratory epithelium. Eur Respir J 2001; 18: 965-970.

14 American Thoracic Society/European Respiratory Society recommendations for standardized procedures for the online and offline measurement of exhaled lower respiratory nitric oxide and nasal nitric oxide, 2005. Am J Respir Crit Care Med 2005; 171: 912-930.

15 Hirst R, Rutman A, Williams G, et al. Ciliated air-liquid cultures as an aid to diagnostic testing of primary ciliary dyskinesia (PCD). Chest 2010; 138: 1441-1447.

16 Cornillie FJ, Lauweryns JM, Corbeel L. Atypical bronchial cilia in children with recurrent respiratory tract infections. A comparative ultrastructural study. Pathol Res Pract 1984; 178: 595-604.

17 Rautiainen M, Nuutinen J, Kiukaanniemi H, et al. Ultrastructural changes in human nasal cilia caused by the common cold and recovery of ciliated epithelium. Ann Otol Rhinol Laryngol 1992; 101: 982-987.

18 Carson JL, Collier AM, Hu SS. Acquired ciliary defects in nasal epithelium of children with acute viral upper respiratory infections. N Engl J Med 1985; 312: 463-468.

19 Kenia P, Rutman A, Williams G, et al. Review of nasal ciliary brushing samples analysed by the diagnostic laboratory for National Primary Ciliary Dyskinesia (PCD) Diagnostic Service. Am J Respir Crit Care Med 2010; 181: A3180.

20 Karadag B, James AJ, Gultekin E, et al. Nasal and lower airway level of nitric oxide in children with primary ciliary dyskinesia. Eur Respir J 1999; 13: 1402-1405.

21 Lundberg JO, Weitzberg E, Nordvall SL, et al. Primarily nasal origin of exhaled nitric oxide and absence in Kartagener's syndrome. Eur Respir J 1994; 7: 1501-1504.

22 Bush A, Cole P, Hariri M, et al. Primary ciliary dyskinesia: diagnosis and standards of care. Eur Respir J 1998; 12: 982-988. 